\title{
Critical conditions and composite Froude numbers for layered flow with transverse variations in velocity
}

\author{
LAR R Y J. PRA T T \\ Woods Hole Oceanographic Institution, Mail Stop 21, 360 Woods Hole Road, \\ Woods Hole, MA, 02543, USA \\ lpratt@whoi.edu
}

(Received 3 December 2007 and in revised form 14 March 2008)

A condition is derived for the hydraulic criticality of a 2-layer flow with transverse variations in both layer velocities and thicknesses. The condition can be expressed in terms of a generalized composite Froude number. The derivation can be extended in order to obtain a critical condition for an $N$-layer system. The results apply to inviscid flows subject to the usual hydraulic approximation of gradual variations along the channel and is restricted to flows in which the velocity remains single-signed within any given layer. For an intermediate layer with a partial segment of sluggish flow, the long-wave dynamics of the overlying and underlying layers become decoupled.

\section{Introduction}

The hydraulic state of a Boussinesq, rigid-lid, two-layer flow is determined by the value of the composite Froude number

$$
G^{2}=F_{1}^{2}+F_{2}^{2}
$$

where $F_{n}=V_{n} /\left(g^{\prime} D_{n}\right)^{1 / 2}, V_{n}$ and $D_{n}$ are the velocity and thickness of layer $n$, and $g^{\prime}$ is the reduced gravity. The flow is considered subcritical, critical, or supercritical, according to $G^{2}<1, G^{2}=1$, or $G^{2}>1$ respectively. Apparently obtained first by Stommel \& Farmer (1952), these criteria apply to a 'slab' flow, meaning that $V_{n}$ and $D_{n}$ are individually uniform in the transverse (cross-stream) direction $(x)$. In geophysical applications, the value of $G^{2}$ is often obtained from data measured along the axis or centreline of a strait (e.g. Armi \& Farmer 1988 for the Strait of Gibraltar), and deductions regarding the presence or lack of hydraulic control and maximal exchange follow. Baines (1995) describes a generalization for the case in which the layer thicknesses vary with $x$, but $V_{1}$ and $V_{2}$ remain constants.

It now appears that progress can be made on the more difficult case in which both $V_{n}$ and $D_{n}$ are allowed to vary with $x$. Moreover, the technique can be applied to a system with an arbitrary number of layers in order to obtain a critical condition, i.e. the condition that the flow supports a free, stationary, linear, long wave. The result is applicable to a number of geophysically important channel flows with transversely varying velocity and depth. Applications include the Strait of Gibralter (e.g. Sannino, Bargagli \& Artale 2002, 2004; Sannino, Carillo \& Artale 2007), the Bosphorus (Gregg \& Özsoy 2002) and Knight Inlet (Klymak \& Gregg 2001). At these sites, the local value of $G^{2}$ can range above and below unity across certain sections, and it is difficult to deduce whether the cross-sectional flow, as a whole, is subcritical, supercritical 
(a)

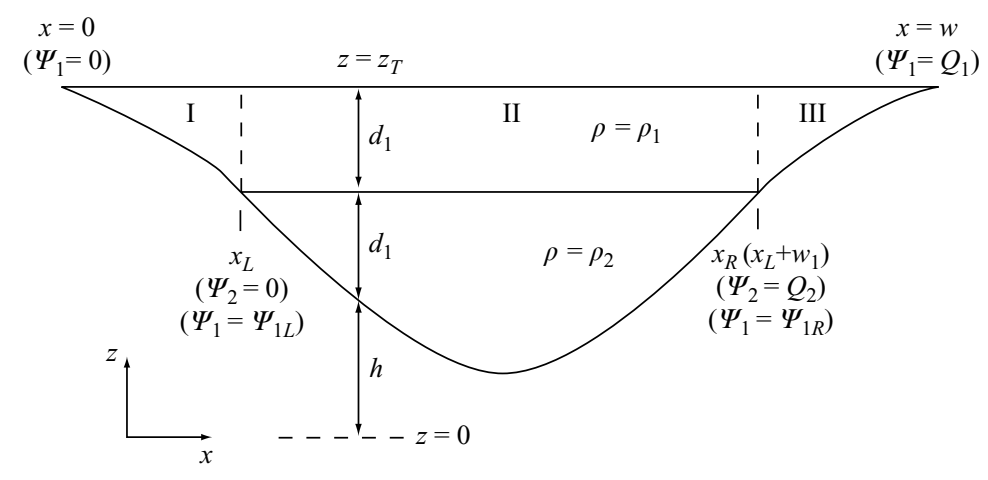

(b)

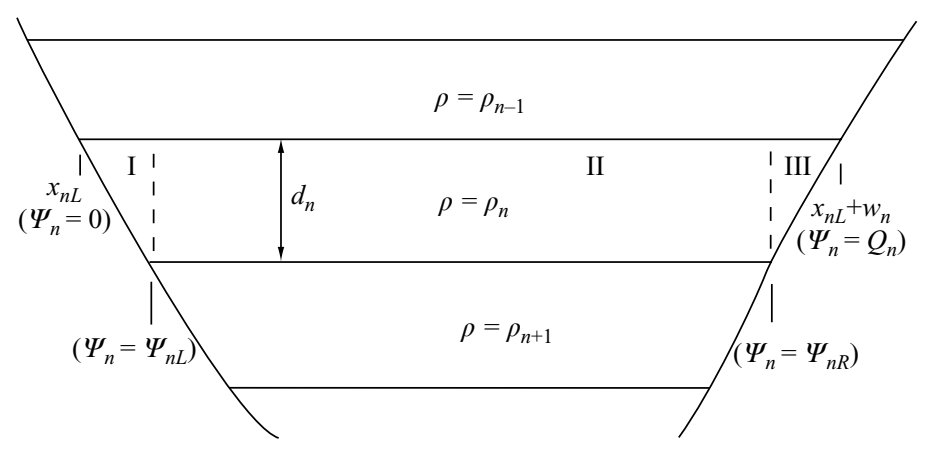

FIGURE 1. Definition sketches for $(a)$ two layers and $(b)$ multiple layers.

or critical. Loosely speaking, critical flow and hydraulic control are associated with a choking mechanism in which upstream effects are produced when the flow is squeezed by its physical boundaries. The upstream effects are carried by a long wave that becomes stationary at the most constricted section. The wave must satisfy the boundary conditions and therefore must have a cross-strait modal structure. The wave speed depends on the flow and topography all across the channel. The results derived herein clarify this dependence.

\section{Two layers}

The methodology is most easily demonstrated for a two-layer system; extension to an arbitrary number of layers is then straightforward. Consider a channel that is aligned in the $y$-direction and for which $x$ represents the transverse direction (figure $1 a$ ). The channel is bounded by a horizontal rigid lid located at elevation $z=z_{T}$ and the bottom elevation $z=h$ is variable. In accordance with the usual 'longwave' approximation of hydraulics, the cross-sectional geometry varies gradually, that is on a scale large compared to the channel depth and width $w$, in the $y$-direction. The channel contains an inviscid, incompressible, two-layer flow, with upper and lowerlayer densities $\rho_{1}$ and $\rho_{2}$. The density difference is slight $0<\left(\rho_{2}-\rho_{1}\right) / \rho_{1} \ll 1$ and the Boussinesq approximation is in effect. Under the assumption of gradual variations along the channel, the $y$-velocity components $v_{1}$ and $v_{2}$ are generally much larger than the $x$-velocities. Both $v_{1}$ and $v_{2}$ are allowed to vary with $x$, though the flow is assumed to be unidirectional ( $v_{n}$ is single-signed and non-zero) within each layer. 
The direction of volume transport in the upper layer may, of course, differ from that of the lower layer. The assumption of gradual variations along the channel and the lack of channel rotation imply that the interface separating the two layers is level and the rigid-lid pressure is independent of $x$. (A formal argument based on long-wave scaling of the $x$-momentum equation appears in Appendix A). The layer thicknesses $d_{1}$ and $d_{2}$ vanish at the edges.

The analysis is based on conservation of energy and volume flux $Q_{n}$ within the two layers:

$$
\begin{array}{r}
B_{1}\left(\psi_{1}\right)=\frac{v_{1}^{2}(x)}{2}+p_{T}, \quad B_{2}\left(\psi_{2}\right)=\frac{v_{2}^{2}(x)}{2}+p_{T}-g^{\prime} d_{1}(x), \\
Q_{1}=\int_{0}^{w} v_{1}(x) d_{1}(x) \mathrm{d} x, \quad Q_{2}=\int_{x_{L}}^{x_{R}} v_{2}(x) d_{2}(x) \mathrm{d} x .
\end{array}
$$

Here $p_{T}$ is the rigid-lid pressure divided by the mean density $\bar{\rho}$, and $g^{\prime}=g\left(\rho_{2}-\rho_{1}\right) / \bar{\rho}$. The Bernoulli function $B_{n}$ for layer $n$ depends only on the streamfunction $\psi_{n}$ for that layer, and the dependence may be regarded as specified by upstream conditions and therefore known. The streamfunction and longitudinal velocity are related by $\partial \psi_{n} / \partial x=v_{n} d_{n}$.

Since the velocity is of single sign in a given layer, the streamfunction $\psi_{n}$ varies monotonically across the channel. At a particular 'section' $y$, each value of $\psi_{n}$ may be assigned a unique position $x_{n}\left(\psi_{n}, y\right)$. (Since further analysis will be carried out at a fixed value of $y$, the $y$ dependence will be suppressed hereafter.) For the upper layer

$$
x_{1}\left(\psi_{1}\right)=\int_{0}^{x_{1}} \mathrm{~d} x=\int_{0}^{\psi_{1}} \frac{\mathrm{d} \psi_{1}^{\prime}}{v_{1} d_{1}},
$$

where the boundary condition $\psi_{1}=0$ has been imposed at the left edge $\left(x_{1}=0\right)$ of the channel. Similarly

$$
x_{2}\left(\psi_{2}\right)-x_{L}=\int_{0}^{\psi_{2}} \frac{\mathrm{d} \psi_{2}^{\prime}}{v_{2} d_{2}},
$$

where $\psi_{2}$ is taken to be zero at the left edge $x=x_{L}$ of the lower layer.

If $(2.1 a, b)$ are used to substitute for the velocities in the above, one obtains

$$
x_{1}\left(\psi_{1}\right)=\int_{0}^{\psi_{1}} \frac{\mathrm{d} \psi_{1}^{\prime}}{2^{1 / 2}\left[B_{1}\left(\psi_{1}^{\prime}\right)-p_{T}\right]^{1 / 2} d_{1}\left(\psi_{1}^{\prime}\right)}
$$

and

$$
x_{2}\left(\psi_{2}\right)-x_{L}=\int_{0}^{\psi_{2}} \frac{\mathrm{d} \psi_{2}^{\prime}}{2^{1 / 2}\left[B_{2}\left(\psi_{2}^{\prime}\right)-p_{T}+g^{\prime} d_{1}\right]^{1 / 2}\left[z_{T}-d_{1}-h\left(x_{2}\left(\psi_{2}^{\prime}\right)\right)\right]},
$$

where the geometrical constraint $\left(d_{2}=z_{T}-d_{1}-h\right)$ has been used in the second result. In the analysis that follows, it will be important to keep in mind that $d_{1}$ varies across the barotropic flange regions (zones I and III in figure $1 a$ ) and is therefore a function of $\psi_{1}$ within those regions. In region II, $d_{1}$ does not depend on $\psi_{1}$. For the lower-layer integral (2.3), $d_{1}$ is uniform but $h$ is variable: $h=h\left(x_{2}\left(\psi_{2}\right)\right)$.

As the channel geometry varies in $y$, the steady flow properties are altered. The functions $B_{1}\left(\psi_{1}\right)$ and $B_{2}\left(\psi_{2}\right)$ remain fixed along the way, as do the layer volume fluxes. As a result of the along-channel evolution, the flow may become hydraulically critical at some section $y$. By definition, the flow at this $y$ is able to support an 
infinitesimal, stationary, long-wave disturbance. The long-wave character implies that the disturbance is subject to the same dynamical constraints as the gradually varying background flow. The existence of a stationary wave means that it is possible to alter the dependent flow variables a slight amount and thereby create a new steady flow satisfying the same upstream conditions. Thus, if

$$
\left(p_{T}, d_{1}\left(\psi_{1}\right), x_{1}\left(\psi_{1}\right), x_{2}\left(\psi_{2}\right), x_{L}\right)=\left(P_{T}, D_{1}\left(\psi_{1}\right), X_{1}\left(\psi_{1}\right), X_{2}\left(\psi_{2}\right), X_{L}\right)
$$

represent the observed flow, which must satisfy (2.2) and (2.3), then the perturbed flow

$$
\left(P_{T}+\tilde{p}_{T}, D_{1}\left(\psi_{1}\right)+\tilde{d}_{1}\left(\psi_{1}\right), X_{1}\left(\psi_{1}\right)+\tilde{x}_{1}\left(\psi_{1}\right), X_{2}\left(\psi_{2}\right)+\tilde{x}_{2}\left(\psi_{2}\right), X_{L}+\tilde{x}_{L}\right)
$$

must also satisfy (2.2) and (2.3) for the same $B_{1}\left(\psi_{1}\right)$ and $B_{2}\left(\psi_{2}\right)$, over the same range of $\psi_{1}$ and $\psi_{2}$, and for the same cross-section geometry $w$ and $h(x)$ A condition for critical flow is that a non-trivial solution for the perturbation exists.

To obtain the required condition, substitute the perturbed fields into (2.2) and (2.3) and linearize the results about the unperturbed state. For the upper layer, the treatment depends on whether the integration takes place over the portion of flange regions of variable layer depth (zones I or III of figure 1a) or in the central region (zone II). In zone I, for example, equation (2.2) becomes

$$
\begin{aligned}
X_{1}\left(\psi_{1}\right)+\tilde{x}_{1}\left(\psi_{1}\right)= & \int_{0}^{\psi_{1}} \frac{\mathrm{d} \psi_{1}^{\prime}}{2^{1 / 2}\left[B_{1}\left(\psi_{1}^{\prime}\right)-P_{T}-\tilde{p}_{T}\right]^{1 / 2}\left[D_{1}\left(X_{1}\left(\psi_{1}^{\prime}\right)\right)+\left(\mathrm{d} D_{1} / \mathrm{d} X_{1}\right) \tilde{x}_{1}\left(\psi_{1}^{\prime}\right)\right]} \\
\cong & \int_{0}^{\psi_{1}} \frac{\mathrm{d} \psi_{1}^{\prime}}{2^{1 / 2}\left[B_{1}\left(\psi_{1}^{\prime}\right)-P_{T}\right]^{1 / 2} D_{1}}+\tilde{p}_{T} \int_{0}^{\psi_{1}} \frac{\mathrm{d} \psi_{1}^{\prime}}{2^{3 / 2}\left[B_{1}\left(\psi_{1}^{\prime}\right)-P_{T}\right]^{3 / 2} D_{1}} \\
& -\int_{0}^{\psi_{1}} \frac{\left(\mathrm{d} D_{1} / \mathrm{d} X_{1}\right) \tilde{x}_{1}\left(\psi_{1}^{\prime}\right)}{2^{1 / 2}\left[B_{1}\left(\psi_{1}^{\prime}\right)-P_{T}\right]^{1 / 2} D_{1}^{2}} \mathrm{~d} \psi_{1}^{\prime} .
\end{aligned}
$$

Next subtract the unperturbed part and replace $2^{1 / 2}\left[B_{1}\left(\psi_{1}^{\prime}\right)-P_{T}\right]^{1 / 2}$ by the unperturbed velocity $V_{1}$ to obtain

$$
\tilde{x}_{1}\left(\psi_{1}\right) \cong \tilde{p}_{T} \int_{0}^{\psi_{1}} \frac{\mathrm{d} \psi_{1}^{\prime}}{V_{1}^{3} D_{1}}-\int_{0}^{\psi_{1}} \frac{\left(\mathrm{d} D_{1} / \mathrm{d} X_{1}\right) \tilde{x}_{1}\left(\psi_{1}\right)}{V_{1} D_{1}^{2}} \mathrm{~d} \psi_{1}^{\prime} .
$$

Differentiation with respect to $\psi_{1}$ leads to

$$
\frac{\mathrm{d} \tilde{x}_{1}}{\mathrm{~d} \psi_{1}}+\frac{\left(\mathrm{d} D_{1} / \mathrm{d} X_{1}\right)}{V_{1} D_{1}^{2}} \tilde{x}_{1} \cong \frac{\tilde{p}_{T}}{V_{1}^{3} D_{1}},
$$

and multiplication of this relation by $D_{1}$ and use of $\mathrm{d} D_{1} / \mathrm{d} X_{1}=\left(\mathrm{d} D_{1} / \mathrm{d} \psi_{1}\right) / V_{1} D_{1}$ lead to

$$
D_{1} \frac{\mathrm{d} \tilde{x}_{1}}{\mathrm{~d} \psi_{1}}+\frac{\mathrm{d} D_{1}}{\mathrm{~d} \psi_{1}} \tilde{x}_{1}=\frac{\mathrm{d}}{\mathrm{d} \psi_{1}}\left(D_{1} \tilde{x}_{1}\right)=\frac{\tilde{p}_{T}}{V_{1}^{3}} \quad \text { (zones I and III). }
$$

The result for zone III is identical.

A similar set of steps can be carried out in zone II, leading to

$$
\frac{\mathrm{d}}{\mathrm{d} \psi_{1}}\left(D_{1} \tilde{x}_{1}\right)=\frac{\tilde{p}_{T}}{V_{1}^{3}}-\frac{\tilde{d}_{1}}{V_{1} D_{1}} \quad \text { (zone II). }
$$

The extra term on the right-hand side (relative to (2.5)) reflects the property that vertical excursions of the interface influence the layer depth in zone II, but not in 
zones I or III. A similar analysis applied in the lower layer leads to

$$
\frac{\mathrm{d}}{\mathrm{d} \psi_{2}}\left(D_{2} \tilde{x}_{2}\right)=\frac{\tilde{p}_{T}}{V_{2}^{3}}+\tilde{d}_{1}\left[\frac{1}{V_{2} D_{2}}-\frac{g^{\prime}}{V_{2}^{3}}\right]
$$

Note that the upper-layer depth perturbation $\tilde{d}_{1}$ is that present in zone II and is therefore independent of $x$.

Equations (2.5)-(2.7) are now integrated across their various zones of applicability and the boundary conditions

$$
D_{1} \tilde{x}_{1}=0\left(\text { at } \psi_{1}=0, Q_{1}\right)
$$

and

$$
D_{2} \tilde{x}_{2}=0\left(\text { at } \psi_{2}=0, Q_{2}\right)
$$

are applied at the layer edges. The upper-layer streamfunction is assumed continuous, with values $\psi_{1 L}$ and $\psi_{1 R}$ at the transitions (figure $1 a$ ). The resulting constraint for the upper layer (obtained by summing the results for zones I, II and II) is

$$
\tilde{p}_{T} \int_{0}^{Q_{1}} \frac{\mathrm{d} \psi_{1}}{V_{1}^{3}}-\tilde{d}_{1} \int_{\psi_{1 L}}^{\psi_{1 R}} \frac{\mathrm{d} \psi_{1}}{V_{1} D_{1}}=0
$$

while the result for the lower layer is

$$
\tilde{p}_{T} \int_{0}^{Q_{2}} \frac{\mathrm{d} \psi_{2}}{V_{2}^{3}}+\tilde{d}_{1} \int_{0}^{Q_{2}}\left(\frac{1}{V_{2} D_{2}}-\frac{g^{\prime}}{V_{2}^{3}}\right) \mathrm{d} \psi_{2}=0
$$

Existence of non-trivial solutions $\left(\tilde{p}_{T}\right.$ and $\left.\tilde{d}_{1}\right)$ to the last two relations requires that

$$
\int_{\psi_{1 L}}^{\psi_{1 R}} \frac{\mathrm{d} \psi_{1}}{V_{1} D_{1}} \int_{0}^{Q_{2}} \frac{\mathrm{d} \psi_{2}}{V_{2}^{3}}+\int_{0}^{Q_{1}} \frac{\mathrm{d} \psi_{1}}{V_{1}^{3}} \int_{0}^{Q_{2}}\left(\frac{1}{V_{2} D_{2}}-\frac{g^{\prime}}{V_{2}^{3}}\right) \mathrm{d} \psi_{2}=0 .
$$

If the $\psi$ integrals are converted back to $x$ integrals, the result can be rearranged to form

$$
G_{w}^{2}=\frac{1}{\frac{1}{w_{I}} \int_{0}^{w} \frac{g^{\prime} D_{1}}{V_{1}^{2}} \mathrm{~d} x}+\frac{1}{\frac{1}{w_{I}} \int_{x_{L}}^{x_{R}} \frac{g^{\prime} D_{2}}{V_{2}^{2}} \mathrm{~d} x}=1 .
$$

Here $w_{I}$ is the width $x_{R}-x_{L}$ of the interface. When the channel cross-section is rectangular and $V_{n}$ are independent of $x, G_{w}$ reduces to the well-known composite Froude number $G$ defined in (1.1). If the cross-section is non-rectangular, $V_{n}$ are constant in each layer, and the cross-section layer areas are $A_{1}$ and $A_{2}$, then (2.8) reduces to

$$
\frac{V_{1}^{2}}{g^{\prime} A_{1} / w_{I}}+\frac{V_{2}^{2}}{g^{\prime} A_{2} / w_{I}}=1
$$

as given by Henderson (1966).

Note that if either layer contains a subsection of sluggish flow $\left|V_{n}^{2} / g^{\prime} d_{n}\right| \ll 1$, this region contributes a very large value to the appropriate denominator in (2.8) and the corresponding term will be $\ll 1$. In this case the condition for critical flow is the same as if the entire layer were inactive. For example, if the upper layer contains a finite segment of stagnant fluid, then the critical condition becomes

$$
w_{I}^{-1} \int_{x_{L}}^{x_{R}} \frac{g^{\prime} D_{2}}{V_{2}^{2}} \mathrm{~d} x=1 .
$$


Related behaviour was pointed out by Stern (1974) in connection with a singlelayer flow in a rotating channel with rectangular cross-section (for which the critical condition is $\left.\int_{0}^{w}\left(V^{2} D\right)^{-1}\left(1-V^{2} / g^{\prime} D\right) \mathrm{d} x=0\right)$ and can also be deduced from the critical condition $g \int_{0}^{d} V^{-2} \mathrm{~d} z=1$ for a homogeneous, free-surface flow with vertical shear (Garrett \& Gerdes 2003). In both cases, a sluggish subinterval makes the entire flow subcritical.

\section{N-layer system}

It is not hard to extend the primary result to a system with $N$ layers (figure $1 b$ ), provided that the width of the channel continuously decreases with depth. The Bernoulli function for the $n$th layer is given by

$$
B_{n}\left(\psi_{n}\right)=\frac{v_{n}^{2}}{2}+p_{T}-\sum_{i=1}^{n-1} g_{n, i}^{\prime} d_{i},
$$

with $g_{n, i}^{\prime}=\left[\left(\rho_{n}-\rho_{i}\right) / \bar{\rho}\right] g$. Then by the same arguments that led to (2.2), the position $x_{n}$ of a streamline $\psi_{n}$ in layer $n$ is given by

$$
x_{n}\left(\psi_{n}\right)-x_{n L}=\int_{0}^{\psi_{n}} \frac{\mathrm{d} \psi_{n}^{\prime}}{2^{1 / 2}\left[B_{n}\left(\psi_{n}^{\prime}\right)-p_{T}+\sum_{i=1}^{n-1} g_{n, i}^{\prime} d_{i}\right]^{1 / 2} d_{n}} .
$$

One now proceeds as before, perturbing the dependent variables $\left(d_{i}\right.$ and $\left.p_{T}\right)$ and requiring that the Bernoulli functions and layer fluxes remain fixed. The steps, which are described in more detail in Appendix B, lead to a set of $N$ simultaneous linear equations for the perturbation thicknesses and lid pressure. This set may be written in the form $\mathbf{a d}=0$, or

$$
\left(\begin{array}{cccccc}
a_{1,1} & 0 & 0 & \cdots & 0 & a_{1, N} \\
a_{2,1} & a_{2,2} & 0 & \cdots & 0 & a_{2, N} \\
a_{3,1} & a_{3,2} & a_{3,3} & 0 & \vdots & a_{3, N} \\
\vdots & \vdots & \vdots & \vdots & 0 & \vdots \\
a_{N-1,1} & a_{N-1,2} & a_{N-1,3} & \cdots & a_{N-1, N-1} & a_{N-1, N} \\
a_{N, 1} & a_{N, 2} & a_{N, 3} & \cdots & a_{N, N-1} & a_{N, N}
\end{array}\right)\left(\begin{array}{c}
\tilde{d}_{1} \\
\tilde{d}_{2} \\
\tilde{d}_{3} \\
\vdots \\
\tilde{d}_{N-1} \\
\tilde{p}_{T}
\end{array}\right)=0,
$$

where

$$
\begin{aligned}
& a_{n, j}=w_{n}-w_{n+1}-g_{n, j}^{\prime} \int_{X_{n L}}^{X_{n R}}\left(\frac{D_{n}}{V_{n}^{2}}\right) \mathrm{d} X_{n} \quad(1 \leqslant n<N, 1 \leqslant j \leqslant n-1), \\
& a_{n, n}=-w_{n+1}(n<N), \quad a_{n, N}=\int_{X_{n L}}^{X_{n R}}\left(\frac{D_{n}}{V_{n}^{2}}\right) \mathrm{d} X_{n} \quad(n \leqslant N), \\
& a_{N, j}=w_{N}-g_{N, j}^{\prime} \int_{X_{N L}}^{X_{N R}}\left(\frac{D_{N}}{V_{N}^{2}}\right) \mathrm{d} X_{N} \quad(1 \leqslant j<N) \quad \text { and } a_{n, j}=0 \quad \text { (otherwise). }
\end{aligned}
$$

The solvability condition for the stationary disturbance $\boldsymbol{d}$ is

$$
\operatorname{det} \mathbf{a}=0
$$


and this provides the critical condition. For example, the $N=3$ result can be written

$$
\tilde{F}_{1}^{2}+\left(\frac{1-r}{r}+\frac{w_{3}}{w_{2}}\right) \tilde{F}_{2}^{2}+\tilde{F}_{3}^{2}-\frac{w_{3}}{w_{2}} \tilde{F}_{1}^{2} \tilde{F}_{2}^{2}-\tilde{F}_{1}^{2} \tilde{F}_{3}^{2}-\frac{1-r}{r} \tilde{F}_{2}^{2} \tilde{F}_{3}^{2}=1
$$

where

$$
\begin{aligned}
& \tilde{F}_{1}^{2}=\left(\frac{1}{w_{2}} \int_{X_{1 L}}^{X_{1 R}} \frac{g_{2,1}^{\prime} D_{1}}{V_{1}^{2}} \mathrm{~d} X_{1}\right)^{-1}, \quad \tilde{F}_{2}^{2}=\left(\frac{1}{w_{2}} \int_{X_{2 L}}^{X_{2 R}} \frac{g_{3,2}^{\prime} D_{2}}{V_{2}^{2}} \mathrm{~d} X_{2}\right)^{-1}, \\
& \tilde{F}_{3}^{2}=\left(\frac{1}{w_{3}} \int_{X_{3 L}}^{X_{3 R}} \frac{g_{3,2}^{\prime} D_{3}}{V_{3}^{2}} \mathrm{~d} X_{3}\right)^{-1},
\end{aligned}
$$

and $r=\left(\rho_{2}-\rho_{1}\right) /\left(\rho_{3}-\rho_{1}\right)$. It can be shown that this expression reduces to Smeed's (2000) result for the three-layer case when the velocities are uniform within each layer and the channel cross-section is rectangular $\left(w_{2}=w_{3}\right)$. An alternative form of the determinantal condition in terms of a tri-diagonal matrix appears in Appendix C.

\section{Discussion}

If the velocity in an intermediate layer $n$ becomes sufficiently small over a finite interval in $x$, then it can be shown (Appendix C) that det a becomes the product of two determinants, one of which involves velocities and depths in the underlying layers and the other velocities and depths in the overlying layers. The critical condition is satisfied by setting either term zero, implying that the overlying layers are decoupled from the underlying layers, at least in terms of the dynamics governing the stationary long wave. As an example, a subsection of nearly stagnant flow in layer 2 of a threelayer system leads to $\tilde{F}_{2}^{2} \rightarrow 0$ in (3.4), which then becomes $\left(\tilde{F}_{1}^{2}-1\right)\left(\tilde{F}_{3}^{2}-1\right)=0$. This condition is satisfied when the upper or lower layer is independently critical: $\tilde{F}_{1}^{2}-1=0$ or $\tilde{F}_{3}^{2}-1=0$. The decoupling of upper- and lower-layer fluid in an $N$-layer system is a generalization of behaviour described by Baines (1995) (also see Engqvist 1996) for the case of uniform layer depths and velocities. The significance of the present result is that long-wave decoupling does not require the separating layer to be completely sluggish, but sluggish only over a sub-interval. If all layers contain intervals of sufficiently sluggish flow, then it can be shown by direct extension of the Appendix B arguments that (3.3) cannot be satisfied; that is, the flow cannot be hydraulically critical. In the three-layer example, $\tilde{F}_{1}^{2}, \tilde{F}_{2}^{2}$ and $\tilde{F}_{3}^{2}$ would all approach zero, precluding satisfaction of (3.4). A physical interpretation is that a flow with sluggish intervals in each layer cannot be choked. Squeezing the flow from the sides does not necessitate change in the volume flux (and therefore the upstream flow) in any layer: rather, the sluggish regions are simply filled in with neighbouring, higher-speed fluid.

The situation is less clear when the velocity changes sign within a given layer. The derivations presented herein, which require a one-to-one relationship between $x$ and $\psi$, are then violated. A stationary wave in the presence of a velocity zero crossing would have a critical line at that location, possibly leading to instability. The significance of 'critical' flow and the implications for hydraulic behaviour in these circumstances is not clear.

Finally, it is not difficult to adapt the critical condition to provide a formula for the phase speed of a neutral long wave in a parallel flow. Consider a long wave mode that propagates along a uniform (in $y$ ) channel with real speed $c$. Since the wave is stationary in a frame of reference moving at the same speed, and in which the apparent layer velocity is $V_{n}-c$, a formula for $c$ is obtained by replacing $V_{n}$ in (3.3) 
(or (2.8)) by $V_{n}-c$. For example, the case of two-layer flow yields

$$
G_{w}^{2}=\frac{1}{\frac{1}{w_{I}} \int_{0}^{w} \frac{g^{\prime} D_{1}}{\left(V_{1}-c\right)^{2}} \mathrm{~d} x}+\frac{1}{\frac{1}{w_{I}} \int_{x_{L}}^{x_{R}} \frac{g^{\prime} D_{2}}{\left(V_{2}-c\right)^{2}} \mathrm{~d} x}=1 .
$$

Again, the coordinate transformation $x \rightarrow \psi$ requires that $V_{n}-c$ be non-zero: the wave may not have a critical line.

Baines (1995, Sec. 4.1) presents a formula for the wave speeds of an $N$-layer system with uniform velocity and thickness in each layer. The polynomial structure of the problem for the wave speed $c$ shows that there are generally $2(N-1)$ waves. Enumeration of the waves of the present system is not so simple. For example, our result (4.1) for $N=2$ is not necessarily quadratic in $c$ and the number of waves is therefore not restricted to 2 .

The previously described decoupling of motion above and below an intermediate layer also applies to wave motion. Suppose that $\left(V_{n}-c\right)^{2}$ becomes $\ll 1$ within a sub-interval of layer $n$; that is, the condition for a critical line $V_{n}\left(x_{c}\right)=c$ in layer $n$ is approached. Then, long-wave motions above and below layer $n$ are decoupled. This result, which is argued formally in Appendix $\mathrm{C}$, appears to be a generalization of the behaviour described by Baines $(1995$, Sec. 4.1$)$ for a layered system with uniform velocity within each layer. There, the presence of a critical layer $\left(V_{n}=c\right)$ causes long-wave motion above and below to decouple.

The work described herein was supported by the Office of Naval Research (N0001407-1-0590) and the National Science Foundation (OCE-0525729).

\section{Appendix A. Justification of the $x$-independent lid pressure and interface elevations}

In formulating the following argument, it may satisfy some reader's intuition to temporarily allow for the presence of rotation about a vertical axis. The cross-channel $(x-)$ momentum equation for an arbitrary layer $(n)$ is then

$$
u_{n} \frac{\partial u_{n}}{\partial x}+v_{n} \frac{\partial u_{n}}{\partial y}-f v_{n}=-\frac{\partial\left(p_{n} / \bar{\rho}\right)}{\partial x}
$$

where $f$ is the Coriolis parameter and where the Boussinesq approximation is again in effect. Tilting of the interfaces, or cross-channel variations of the rigid-lid pressure, are reflected in the pressure gradient term and we wish to estimate this term.

In a hydraulically driven flow, one expects the along-channel velocity $v_{n}$ to scale with the internal wave speed, which can roughly be estimated as $\left(g^{\prime} D\right)^{1 / 2}$, where $g^{\prime}$ and $D$ are representative values of reduced gravity and depth. The hydrostatic relation suggests that $p_{n} / \rho_{o}$ itself scales with $g^{\prime} D$. The standard hydraulic assumption of gradual variations along the path of the flow means that the cross-channel length scale $W$, often just the channel width, is much less than the along-channel length scale $L$. With this scale mismatch, the continuity equation for layer $n$ suggest $(W / L)$ $\left(g^{\prime} D\right)^{1 / 2}$ as a scale for the cross-channel velocity. Reformulation of (A1) in terms of 
non-dimensional variables (with tildes) based on these scales leads to

$$
\left(\frac{W}{L}\right)^{2}\left[\tilde{u}_{n} \frac{\partial \tilde{u}_{n}}{\partial \tilde{x}}+\tilde{v}_{n} \frac{\partial \tilde{u}_{n}}{\partial \tilde{y}}\right]-\frac{f W}{\left(g^{\prime} D\right)^{1 / 2}} \tilde{v}_{n}=-\frac{\partial \tilde{p}_{n}}{\partial \tilde{x}} .
$$

In the limit $(W / L \rightarrow 0)$ of gradual variations, which applies to all traditional hydraulic models, cross-channel pressure gradients are due entirely to the Coriolis acceleration acting on the along-channel flow. This is the situation in hydraulic models of rotating channel flow (e.g. Gill 1977), where density interfaces tilt in the $x$-direction. Flows that lack rotation cannot support such gradients since that would require fluid acceleration in the $x$-direction. The assumed small horizontal aspect ratio $(W / L)$ does not permit this.

\section{Appendix B. Treatment of the case of $N$ layers, with $N>2$}

Since the width of the channel continuously decreases with depth, each layer (save the bottom layer) has flange regions (I and III in figure $1 b$ ) that contact the bottom, along with a middle region (II) that has uniform thickness. In Region I of such a layer, the departure $\tilde{x}_{n}$ in the position $x_{n}\left(\psi_{n}\right)$ of streamline $\psi_{n}$ about its background position $X_{n}\left(\psi_{n}\right)$ is given by

$$
\begin{aligned}
\tilde{x}_{n}\left(\psi_{n}\right)-\tilde{x}_{n}(0)= & \left(\tilde{p}_{T}-\sum_{i=1}^{n-1} g_{n, i}^{\prime} \tilde{d}_{i}\right) \int_{0}^{\psi_{n}} \frac{\mathrm{d} \psi_{n}^{\prime}}{V_{n}^{3} D_{n}} \\
& +\int_{0}^{\psi_{n}} \frac{1}{V_{n} D_{n}^{2}}\left(\sum_{i=1}^{n-1} \tilde{d}_{i}-\frac{\mathrm{d} D_{n}}{\mathrm{~d} x_{n}} \tilde{x}_{n}\right) \mathrm{d} \psi_{n}^{\prime} \quad(n<N),
\end{aligned}
$$

where $V_{n}$ and $D_{n}$ represent the undisturbed velocity and layer thickness and $\tilde{d}_{n}\left(\psi_{n}\right)$ the perturbation thickness. The steps that lead from (3.1) to (B1) are similar to those used to obtain (2.4) from (2.2). Differentiation with respect to $\psi_{n}$ leads, after some rearrangement, to

$$
\frac{\mathrm{d}}{\mathrm{d} \psi_{n}}\left(D_{n} \tilde{x}_{n}\right)=\frac{\left(\tilde{p}_{T}-\sum_{i=1}^{n-1} g_{n, i}^{\prime} \tilde{d}_{i}\right)}{V_{n}^{3}}+\frac{\sum_{i=1}^{n-1} \tilde{d}_{i}}{V_{n} D_{n}}(n<N) .
$$

The same result holds in region III.

In zone II, the development is similar to that used to obtain (2.6) and results in

$$
\frac{\mathrm{d}}{\mathrm{d} \psi_{n}}\left(D_{n} \tilde{x}_{n}\right)=\frac{\tilde{p}_{T}-\sum_{i=1}^{n-1} g_{n, i}^{\prime} \tilde{d}_{i}}{V_{n}^{3}}-\frac{\tilde{d}_{n}}{V_{n} D_{n}} .
$$

If (B2) and (B3) are integrated across their zones of applicability, the values of $D_{n} \tilde{x}_{n}$ are matched at the joining edges, and the resulting $\psi_{n}$ integrals are converted to $X_{n}$ integrals, one obtains

$$
\tilde{p}_{T} \int_{X_{n L}}^{X_{n R}} \frac{D_{n}}{V_{n}^{2}} \mathrm{~d} X_{n}-w_{n+1} \sum_{i=1}^{n} \tilde{d}_{i}+\sum_{i=1}^{n-1} \tilde{d}_{i}\left[w_{n}-g_{n, i}^{\prime} \int_{X_{n L}}^{X_{n R}}\left(\frac{D_{n}}{V_{n}^{2}}\right) \mathrm{d} X_{n}\right]=0
$$


The bottom layer (layer $N$ ) can be treated in a manner analogous to that for the two-layer system, resulting in

$$
\tilde{p}_{T} \int_{X_{N L}}^{X_{N R}} \frac{D_{N}}{V_{N}^{2}} \mathrm{~d} X_{N}+\sum_{i=1}^{N-1} \tilde{d}_{i}\left[w_{N}-g_{N, i}^{\prime} \int_{X_{N L}}^{X_{N R}}\left(\frac{D_{N}}{V_{N}^{2}}\right) \mathrm{d} X_{N}\right]=0 .
$$

Together (B4) and (B5) form a set of $N$ simultaneous linear equations for the perturbation layer thicknesses and lid pressure, as summarized in (3.2).

\section{Appendix C. Long-wave decoupling and critical layers}

For purposes of taking the determinant, the $N \times N$ matrix a (3.2) can be transformed to the $(N-1) \times(N-1)$ tridiagonal matrix

$$
\left(\begin{array}{cccccc}
g_{2,1}^{\prime}-\frac{w_{2}}{I_{1}}-\frac{w_{2}}{I_{2}} & \frac{w_{3}}{I_{2}} & 0 & \cdots & 0 & 0 \\
\frac{w_{2}}{I_{2}} & g_{3,2}^{\prime}-\frac{w_{3}}{I_{2}}-\frac{w_{3}}{I_{3}} & \frac{w_{4}}{I_{3}} & \cdots & 0 & 0 \\
0 & \frac{w_{3}}{I_{3}} & g_{4,3}^{\prime}-\frac{w_{4}}{I_{3}}-\frac{w_{4}}{I_{4}} & 0 & \vdots & 0 \\
\vdots & \vdots & \vdots & \vdots & \vdots & \vdots \\
0 & 0 & 0 & \cdots & g_{N-1, N-2}^{\prime}-\frac{w_{N-1}}{I_{N-2}}-\frac{w_{N-1}}{I_{N-1}} & \frac{w_{N}}{I_{N-1}} \\
0 & 0 & 0 & \cdots & \frac{w_{N-1}}{I_{N-1}} & g_{N, N-1}^{\prime}-\frac{w_{N}}{I_{N-1}}-\frac{w_{N}}{I_{N}}
\end{array}\right)
$$

where

$$
I_{n}=\int_{X_{n L}}^{X_{n R}}\left(\frac{D_{n}}{V_{n}^{2}}\right) \mathrm{d} x
$$

The transformation begins with division of each row $n$ of a by $a_{n, N}$. Each row $n$ $(n>1)$ is then subtracted from row $n-1$, and the corresponding differences are used to form a new matrix of dimension $N-1$. This step removes the rigid lid pressure perturbation (and corresponding barotropic motions) from consideration. The new matrix is then converted into the form (C1) by subtracting each column $n$ from $n-1$ but leaving the final column intact. The determinant is unchanged by this procedure and the critical condition is obtained by setting it to zero. As described in $\S 4$, the speeds of a set of neutral long waves can be obtained by replacing $I_{n}$ by

$$
\int_{X_{n L}}^{X_{n R}}\left(\frac{D_{n}}{\left(V_{n}-c\right)^{2}}\right) \mathrm{d} x
$$

The matrix (C1) is similar in form to that described by Baines (1995, eq. 4.1.8) for a layered system with uniform velocity within each layer and with a rectangular cross-section. Similar deductions follow concerning decoupling of layers. In particular, if one of the intermediate layers moves at the critical speed $V_{n}=c$ for a long wave, then the corresponding terms in the matrix become zero. In this case it can be shown that the layers above and below layer $n$ become decoupled.

An analogous situation occurs in our problem with non-uniform layer velocities. Suppose that in layer $n,\left(V_{n}(x)-c\right)^{2}$ becomes sufficiently small in some $x$ interval that terms like $w_{n} / I_{n}$ become much smaller than terms like $g_{n, n-1}^{\prime}$ in the same row of the matrix. (In the case of hydraulically critical flow $(c=0)$ the situation arises when $V_{n}^{2}$ becomes sufficiently small.) If the terms invoving $1 / I_{n}$ are set to zero, it follow from simple inspection that the determinant of the full matrix (C1) becomes the product 
of two co-determinants:

$$
\begin{aligned}
\operatorname{det}\left(\begin{array}{ccc}
g_{2,1}^{\prime}-\frac{w_{2}}{I_{1}}-\frac{w_{2}}{I_{2}} & \frac{w_{3}}{I_{2}} & 0 \\
\frac{w_{2}}{I_{2}} & g_{3,2}^{\prime}-\frac{w_{3}}{I_{2}}-\frac{w_{3}}{I_{3}} & \vdots \\
0 & \cdots & g_{n, n-1}^{\prime}-\frac{w_{n}}{I_{n-1}}
\end{array}\right) \\
\quad \times \operatorname{det}\left(\begin{array}{ccc}
g_{n+1, n}^{\prime}-\frac{w_{n+1}}{I_{n+1}} & \frac{w_{n+2}}{I_{n+1}} & 0 \\
\frac{w_{n+1}}{I_{n+1}} & g_{n+2, n+1}^{\prime}-\frac{w_{n+2}}{I_{n+1}}-\frac{w_{n+2}}{I_{n+2}} & \vdots \\
0 & \cdots & g_{N-1, N-2}^{\prime}-\frac{w_{n-1}}{I_{n-1}}
\end{array}\right)=0
\end{aligned}
$$

The first matrix involves velocities and layer depths above layer $n$; the second involves velocities and layer depths below. If the first determinant is zero while the second is not, then the depth perturbations $\tilde{d}_{i}$ are finite in the layers overlying $n$ but are zero in the underlying layers. In this case, layer $n$ and the fluid beneath acts like an inactive fluid or, alternatively, like a perfect reflector of overlying long-wave motion.

\section{REFERENCES}

ARmi, L. \& FARMER, D. M. 1988 The flow of Mediterranean water through the Strait of Gibraltar. Prog. Oceanogr. 21, 1-105.

BaInes, P. G. 1995 Topographic Effects in Stratified Flows. Cambridge University Press.

ENGQVIST, A. 1996. Self-similar multi-layer exchange flow through a contraction. J. Fluid Mech. 328, 49-66.

Garrett, C. \& Gerdes, F. 2003 Hydraulic control of homogeneous shear flows. J. Fluid Mech. 475, $163-172$.

GiLl, A. E. 1977 The hydraulics of rotating-channel flow. J. Fluid Mech. 80, 641-671.

GregG, M. \& Özsoy, E. 2002 Flow, water mass changes, and hydraulics in the Bosphorus. $J$. Geophys. Res. 107, 3016.

Henderson, F. M. 1966 Open Channel Flow. Macmillan.

Klymak, J. M. \& GregG, M. C. 2001 Three-dimensional nature of flow near a sill. J. Geophys. Res. 106, 22295-22311.

Sannino, G., Bargagli, A. \& Artale, V. 2002 Numerical modeling of the mean exchange through the Strait of Gibraltar. J. Geophys. Res. 107, 3094.

Sannino, G., Bargagli, A. \& Artale, V. 2004 Numerical modeling of the semidiurnal tidal exchange through the Strait of Gibraltar. J. Geophys. Res. 109, doi:10.1029/2003JC002057.

Sannino, G., Carillo, A. \& Artale, V. 2007 Three-layer view of transports and hydraulics in the Strait of Gibraltar: A three-dimensional model study. J. Geophys. Res. 112, doi:10.1029/2006JC003717.

SmeED, D. A. 2000 Hydraulic control of three-layer exchange flows: application to the Bab al Mandab. J. Phys. Oceanogr. 30, 2574-2588.

Stern, M. E. 1974 Comment on rotating hydraulics. Geophys. Fluid Dyn. 6, 127-130.

Stommel, H. M. \& Farmer, H. G. 1952 Abrupt change in width in two-layer open channel flow. $J$. Mari. Res. 11, 205-214. 